ISSN 0103-5150

Fisioter. Mov., Curitiba, v. 25, n. 1, p. 31-45, jan./mar. 2012 Licenciado sob uma Licença Creative Commons

\title{
Relação entre a Avaliação da Coordenação e Destreza Motora (Acoordem) e a Classificação Internacional de Funcionalidade, Incapacidade e Saúde (CIF)
}

\author{
Linking the Motor Coordination and Dexterity Assessment (MCDA) to the \\ International Classification of Functioning, Disability and Health (ICF)
}

\author{
Ana Amélia Cardoso, ${ }^{[a]}$ Lívia de Castro Magalhães, ${ }^{[b]}$ Tatiana Teixeira Barral de Lacerda, ${ }^{[c]}$ \\ Peterson Marco de Oliveira Andrade ${ }^{[d]}$ \\ [a] Terapeuta ocupacional, Doutoranda em Ciências da Reabilitação pela Universidade Federal de Minas Gerais (UFMG), \\ professora assistente do Departamento de Terapia Ocupacional, Universidade Federal do Paraná (UFPR), Curitiba, PR - \\ Brasil, e-mail: anaameliato@yahoo.com.br \\ [b] Terapeuta ocupacional, Doutora em Educação, professora titular do Departamento de Terapia Ocupacional, Universidade \\ Federal de Minas Gerais (UFMG), Belo Horizonte, MG - Brasil, e-mail: liviam@gcsnet.com.br \\ [c] Fisioterapeuta, Mestre em Ciências da Reabilitação pela Universidade Federal de Minas (UFMG), professora do curso \\ de Fisioterapia da Pontifícia Universidade Católica de Minas Gerais (PUC -Minas), Belo Horizonte, MG - Brasil, e-mail: \\ tatianabarral@terra.com.br \\ [d] Fisioterapeuta, Doutor em Neurociências pela Universidade Federal de Minas Gerais (UFMG), professor adjunto do \\ Departamento de Fisioterapia da Universidade Federal dos Vales do Jequitinhonha e Mucuri, Diamantina, MG - Brasil, \\ e-mail: peterson@ufvjm.edu.br
}

\section{Resumo}

Introdução: A Classificação Internacional de Funcionalidade, Incapacidade e Saúde (CIF) visa a proporcionar uma base científica para a compreensão e o estudo da saúde e das condições relacionadas, podendo ser utilizada para nortear a criação e utilização de medidas de desfecho em reabilitação. A Avaliação da Coordenação e Destreza Motora (Acoordem) é um teste brasileiro, criado para detectar atraso motor em crianças de 4 a 8 anos. Objetivos: Os objetivos deste estudo foram: a) associar as subcategorias da CIF aos itens da Acoordem; e b) determinar se os itens da Acoordem se encaixam na estrutura da CIF. Materiais e métodos: Uma terapeuta ocupacional e um fisioterapeuta codificaram independentemente os itens contidos na Acoordem, fazendo o linking com a CIF. Para os itens em que não houve concordância entre os pesquisadores, foi usada a opinião de outras duas pesquisadoras, uma terapeuta ocupacional e uma fisioterapeuta. Resultados: Os itens da Acoordem foram associados aos componentes $b$ (funções do corpo), $d$ (atividades 
e participação), e (fatores ambientais) e $f$ (fatores pessoais). Não houve itens relacionados com o componente $s$ (estrutura do corpo) e apenas três itens foram codificados como nc (não coberto pela CIF). Discussão e conclusão: Os itens da Acoordem puderam ser relacionados à estrutura da CIF. Profissionais e pesquisadores da área de reabilitação podem usar a Acoordem para obter dados acerca da atividade e participação em crianças com problemas de coordenação motora, pois sua terminologia e abrangência está de acordo com a perspectiva biopsicossocial da OMS.

Palavras-chave: CIF. Avaliação de desempenho. Avaliação da coordenação. Destreza motora.

\section{Abstract}

Introduction: The International Classification of Functioning, Disability and Health (ICF) aims to provide a scientific basis for the comprehension and the study of health and its related conditions, and as such it can be used to guide the creation and utilization of outcome measures. The Motor Coordination and Dexterity Assessment (MCDA) is a Brazilian assessment tool that was designed to detect motor delay in 4 to 8 years old children. Objectives: The aims of this study were: a) to link the CIF sub domains with the MCDA items and b) determine if the MCDA items fit with the ICF framework. Materials and methods: One occupational therapist and one physical therapist coded, independently, the MCDA items, conducting the linking with the ICF. Whenever there was no agreement between the researchers, the opinion of other two researchers, one occupational therapist and one physical therapist, was used. Results: The MCDA items were linked to the components b (body functions), d (activities and participation), e (environmental factors), and $\mathrm{f}$ (personal factors). Discussion and conclusion: The MCDA items could be linked with the ICF framework. Professionals and researchers in the rehabilitation field can use the MCDA to get data about activities and participation of children with motor coordination problems, because the MCDA terminology and scope are in accordance with WHO's biopsychosocial perspective.

Keywords: ICF. Performance assessment. Motor coordination. Dexterity assessment.

\section{Introdução}

A "Família de Classificações Internacionais" da Organização Mundial de Saúde (OMS) visa a estabelecer uma linguagem comum para a descrição da saúde em seus vários campos em todo o mundo. As classificações facilitam o levantamento, a consolidação, a análise e a interpretação dos resultados, a formação de bases de dados consistentes, e permitem a comparação de informações entre populações de regiões e países ao longo do tempo (1).

A Classificação Estatística Internacional de Doenças e Problemas Relacionados à Saúde, conhecida como CID, classifica as condições de saúde, transtornos ou lesões, fornecendo um modelo baseado na etiologia, anatomia e causas externas das lesões $(1,2)$. Atualmente, a CID-10 é a classificação diagnóstica padrão internacional para propósitos epidemiológicos gerais e administrativos da saúde, incluindo análise da situação geral da saúde de grupos populacionais e o monitoramento da incidência e prevalência de doenças e outros problemas de saúde $(3,4)$.

Enquanto a CID-10 é usada para classificar mortalidade e morbidade, a Classificação Internacional de Funcionalidade, Incapacidade e Saúde (CIF) classifica as condições de saúde (5). A CIF representa uma mudança de paradigma para se pensar e trabalhar a deficiência e a incapacidade, constituindo um instrumento importante para avaliação da qualidade de vida e para a promoção de políticas de inclusão social (1). No modelo da CIF, a incapacidade não é definida apenas pela lesão ou doença, mas considerada como resultante da interação entre a disfunção (orgânica e/ou da estrutura do corpo) apresentada pelo indivíduo, a limitação de suas atividades e a restrição na participação social, bem como dos fatores ambientais, que podem atuar como barreiras ou facilitadores para o desempenho $(1,5-7)$.

A CIF tem como objetivo geral "proporcionar uma linguagem unificada e padronizada e uma estrutura que descreva a saúde e os estados relacionados 
à saúde" (6). A CIF visa a fornecer uma base científica para a compreensão e o estudo da saúde e das condições relacionadas à saúde, por meio de seus determinantes e efeitos. Sua linguagem padronizada busca facilitar a comunicação entre profissionais, pesquisadores, elaboradores de políticas públicas e o público. Além disso, a CIF fornece um sistema de codificação para sistemas de informação, permitindo a comparação de dados entre países e serviços de saúde em diferentes momentos. (8). A OMS estimula a utilização da CIF em diversas áreas, como saúde, educação, previdência social, medicina do trabalho, estatísticas e políticas públicas (1, 3, 6, 9-11).

Em 2004, a OMS publicou uma versão da CIF para crianças e jovens (12), uma vez que a experiência de saúde e incapacidade para crianças e jovens não é a mesma que para os adultos (13). Alguns subdomínios da CIF tinham apenas relevância indireta para crianças e havia omissões importantes relevantes para a infância e adolescência (14), sendo as áreas de particular importância aquelas que refletiam progresso no desenvolvimento nos primeiros anos da infância (15). Por isso, a versão da CIF para crianças e jovens (12) inclui subdomínios, como explorar objetos com a boca (d1200), e descrições modificadas de domínios, como apropriado para a idade (14).

A OMS aponta que a CID-10 e a CIF são complementares, pois a informação sobre mortalidade e morbidade, fornecida pela CID-10, e a informação sobre a saúde e evolução relacionada à saúde, fornecida pela CIF, podem ser combinadas em medidas-resumo da saúde da população. Os usuários são incentivados a usá-las em conjunto, a fim de obter um quadro mais amplo sobre a saúde do indivíduo ou populações $(1,2,6,16,17)$.

Uma característica fundamental de um sistema de classificação é a sua capacidade de servir como estrutura para o desenvolvimento de medidas e recursos de avaliação que reflitam as dimensões específicas daquela classificação (15). A escolha da CID-10 ou da CIF como modelo irá influenciar o desenvolvimento do instrumento, bem como o tipo de informação que será obtida por meio de sua aplicação.

Na área de reabilitação, instrumentos padronizados de avaliação podem ser usados para avaliar os problemas do indivíduo, para estabelecer metas e avaliar efeitos da intervenção (18). Grande parte dos instrumentos e medidas disponíveis, no entanto, informa principalmente sobre a patologia e seu impacto em órgãos e sistemas do corpo do indivíduo $(2,18)$, o que sugere que são instrumentos norteados pela CID-10. Ao usar a CID-10 como modelo norteador para a criação de um instrumento, o foco do teste seria apenas no diagnóstico ou descoberta dos sintomas relacionados a uma condição patológica (2). Por outro lado, se o objetivo do teste é obter, além do diagnóstico clínico, informação a respeito das consequências funcionais que uma doença traz para a vida de um indivíduo, a CIF seria um modelo norteador mais interessante, uma vez que enfatiza as repercussões da doença no cotidiano do indivíduo $(2,18)$.

Dada a relevância da perspectiva da CIF para a área de reabilitação, observam-se esforços no sentido de criar recursos de avaliação congruentes com o modelo proposto, além de estudos para verificar a consistência entre os testes existentes e a CIF. Nesse sentido, Cieza et al. (19) propõem um procedimento padronizado para verificar se os itens de um instrumento apresentam relação clara com os componentes da CIF e orientar a codificação dos itens de acordo com os componentes da classificação. Ao criar um novo instrumento usando a CIF como modelo norteador, é interessante que o autor utilize as regras propostas por Cieza et al. (19), a fim de garantir que o teste realmente se encaixa no modelo proposto.

De acordo com Di Nubila (3), as avaliações funcionais não podem continuar sendo realizadas apenas com o olhar médico, biológico e focado no corpo, quando a CIF se apresenta como ferramenta mais adequada e abrangente. A tendência atual é que a CIF se torne a base para a avaliação multiprofissional do cliente, definição de metas, gerenciamento de intervenção e medida de resultados (18). Diferentes estudos utilizaram as regras de linking para identificar e comparar com a CIF os conceitos contidos em instrumentos de avaliação genéricos e específicos de doenças crônicas (20-22). Além disso, as regras de linking foram usadas para categorizar a percepção dos pacientes sobre o impacto da doença e para validar os core sets da CIF $(23,24)$.

Fisioterapeutas e terapeutas ocupacionais que atuam na área infantil lidam com crianças com diferentes graus de comprometimento motor, sendo importante contar com instrumentos adequados para identificar crianças que necessitam de intervenção. A Avaliação da Coordenação e Destreza Motora (Acoordem) (25) é um teste criado para oferecer aos profissionais brasileiros, que lidam com crianças de 4 a 8 anos, um instrumento válido, confiável, de 
fácil aplicação e baixo custo para detecção de atraso motor. É importante enfatizar que a criação da Acoordem não objetiva solucionar todos os problemas relacionados à avaliação e identificação do TDC, mas, considerando as tendências da literatura atual, ela se enquadra na perspectiva de avaliar os componentes motores em associação com habilidades funcionais relevantes para o desempenho em casa e na escola, como proposto no modelo da CIF (6). 0 teste conta com itens para avaliação das habilidades sensório-motoras, por meio da observação de itens puramente motores como força, equilíbrio e coordenação motora; além da avaliação do desempenho funcional em casa e na escola e das preferências no brincar, por meio dos questionários de pais e professores.

A Acoordem foi criada com base em extensiva revisão da literatura e se caracterizando como avaliação de produto final, do que é observável (ações da criança), e não do porquê da incapacidade (26). A Acoordem é um teste descritivo ou discriminativo $(27,28)$, cujo objetivo é classificar, categorizar ou descrever a criança. 0 teste contém itens para avaliar o desempenho motor da criança em três áreas: coordenação e destreza manual, coordenação corporal e planejamento motor e desempenho em casa e na escola (questionários de pais e professores). Vários estudos (29-32) foram realizados para verificar a validade de conteúdo e as qualidades psicométricas dos itens de cada área, separadamente, sendo verificada a confiabilidade teste reteste/entre examinadores e a discriminação de desempenho por idade, possibilitando a definição dos itens que compõem a versão atual do instrumento (25).

Tendo em vista que a Acoordem é um instrumento novo, ainda em fase de validação, cuja criação foi norteada pelo modelo da CIF (6), o objetivo do presente estudo foi aplicar o grupo de regras propostas por Cieza et al. (19) para relacionar a CIF aos itens da ACOORDEM e determinar se tais itens de fato se encaixam na estrutura da CIF.

\section{Materiais e métodos}

O sistema de classificação da CIF é hierárquico, dividido em duas partes. A primeira parte se refere à funcionalidade e incapacidade e possui dois componentes: 1) funções e estrutura do corpo; e 2) atividades e participação. Na segunda parte, fatores contextuais, estão os fatores ambientais e os fatores pessoais $(4,6,9,33)$. A unidade de classificação da CIF são categorias dentro dos domínios de saúde e daqueles relacionados à saúde; a CIF não classifica pessoas, mas descreve a situação de cada pessoa dentro de uma gama de domínios de saúde ou relacionados à saúde, sempre dentro do contexto dos fatores ambientais e pessoais (6).

A codificação da CIF usa um sistema alfanumérico com uma letra para nomear o componente ao qual o item se refere: $b$ (funções do corpo), $s$ (estruturas do corpo), $d$ (atividades e participação), e $e$ (fatores ambientais). Na sequência, é adicionado um código numérico com dois, três ou quatro dígitos, referente à categoria individual do referido componente. $(4,6,34,35)$.

No presente estudo foi usada a versão experimental da Acoordem (25). Uma terapeuta ocupacional (AAC) e um fisioterapeuta (PMOA) desempenharam independentemente o linking entre a CIF e os itens contidos na Acoordem. Para os itens específicos do desenvolvimento infantil, o linking foi feito com a versão da CIF para crianças e jovens (12). Para os itens em que não houve concordância entre os pesquisadores, foi usada a opinião de outras duas pesquisadoras, uma terapeuta ocupacional e uma fisioterapeuta (LCM e TTBL).

$\mathrm{O}$ procedimento de linking foi realizado independentemente, seguindo as regras padronizadas por Cieza et al. (19). Todos os pesquisadores tinham conhecimento prévio sobre o conteúdo teórico e taxonômico da CIF. De acordo com as regras, cada conceito contido nos itens do instrumento deve ser correlacionado à mais precisa categoria da CIF. Uma tabela com as oito regras propostas pode ser encontrada em Cieza et al. (19).

Itens com conceitos insuficientes para decidir qual componente da CIF representa melhor devem ser classificados pelas letras "nd" (não definível); quando o item se refere a um estado de saúde, ele deve ser registrado como nd-sg; nd-sf; nd-sm; nd-qv (não definível - saúde geral; não definível - saúde física; não definível - saúde mental; não definível qualidade de vida, respectivamente) (19). Itens que consideram claramente fatores pessoais, que não estão codificados pela CIF, devem ser marcados com as letras "fp" (fator pessoal). Conceitos que não são cobertos pela CIF devem ser marcados com as letras "nc" (não coberto), e conceitos relacionados ao diagnóstico ou condição de saúde são identificados com as letras "cs" - condição de saúde (19). 


\section{Resultados}

Os itens de observação direta da Acoordem foram relacionados a 15 categorias, de seis capítulos da CIF: quatro categorias pertencem ao componente $b$ (funções do corpo), e 11 ao componente $d$ (atividades e participação). No componente $b$ (funções do corpo), os itens foram correlacionados a categorias nos capítulos 1 (Funções mentais); 2 (Funções sensoriais e dor); e 7 (Funções neuromusculoesqueléticas e relacionadas ao movimento), enquanto no componente $d$ (atividades e participação), foi feita correlação com categorias dos capítulos 1 (Aprendizagem e aplicação do conhecimento); 4 (Mobilidade); e 9 (Vida comunitária, social e cívica). A maior parte dos itens de observação da Acoordem foi correlacionada a categorias do capítulo 4 do componente $d$ (atividades $e$ participação). Não houve itens codificados com os componentes $s$ (estrutura do corpo) e $e$ (fatores ambientais). A codificação completa é apresentada no Quadro 1.

Ao considerar o Questionário de Pais da Acoordem, os 90 itens foram associados a 52 categorias, de 12 capítulos da CIF, dos componentes $b$ (funções do corpo), $d$ (atividades e participação) e $e$ (fatores

Quadro 1 - Codificação dos itens de observação direta da criança de acordo com a CIF

\begin{tabular}{|c|c|c|}
\hline Componente da CIF & Categoria da CIF & Item da Acoordem \\
\hline \multirow{12}{*}{ Funções do corpo } & b176 (funções mentais para & Jogo da batucada: batendo cubos \\
\hline & $\begin{array}{l}\text { a sequência de movimentos } \\
\text { complexos) }\end{array}$ & Labirintos 1 e 2 \\
\hline & \multirow{4}{*}{$\begin{array}{l}\text { b2351 (função vestibular do } \\
\text { equilíbrio) }\end{array}$} & Equilíbrio um pé só: olhos abertos perna direita \\
\hline & & Equilíbrio um pé só: olhos abertos perna esquerda \\
\hline & & Equilíbrio um pé só: olhos fechados perna direita \\
\hline & & Equilíbrio um pé só: olhos fechados perna esquerda \\
\hline & \multirow{2}{*}{$\begin{array}{l}\text { b740 (funções da resistência } \\
\text { muscular) }\end{array}$} & Padrão de extensão em prono \\
\hline & & Padrão de flexão em supino \\
\hline & \multirow{4}{*}{$\begin{array}{l}\text { b7602 (coordenação de } \\
\text { movimentos voluntários) }\end{array}$} & Prova polegar dedos \\
\hline & & Traçado simples: percurso reto, circular \\
\hline & & Traçado complexo: trilha da borboleta \\
\hline & & Cópia de figuras geométricas \\
\hline \multirow{14}{*}{ Atividades e participação } & d130 (imitar) & Cópia de sentença \\
\hline & d145 (aprender a escrever) & Escrever alfabeto: letra cursiva \\
\hline & d4400 (pegar) & Colocar cinco moedas no cofre \\
\hline & \multirow[t]{11}{*}{ d4401 (agarrar) } & Colocar cinco moedas no cofre \\
\hline & & Distribuir 20 cartas \\
\hline & & Recorte de quadrado e círculo \\
\hline & & Recorte do gato \\
\hline & & Colocar pinos na tábua: tábua com 12 furos \\
\hline & & $\begin{array}{l}\text { Mudar pinos de fileira, fazendo rotação do pino de duas cores } \\
\text { entre os dedos }\end{array}$ \\
\hline & & Pesponto na tábua de seis furos, fio flexível de $60 \mathrm{~cm}$, de ponta dura \\
\hline & & Colocar cinco moedas no cofre \\
\hline & & Distribuir 20 cartas \\
\hline & & Recorte de quadrado e círculo \\
\hline & & Recorte do gato \\
\hline
\end{tabular}


Quadro 1 - Codificação dos itens de observação direta da criança de acordo com a CIF

(Conclusão)

\begin{tabular}{lll}
\hline Componente da CIF & Categoria da CIF & Item da Acoordem \\
\hline d4403 (soltar) & Colocar cinco moedas no cofre \\
& Distribuir 20 cartas \\
\hline d4454 (atirar) & Repicar bola de $20 \mathrm{~cm}$ no chão e agarrar com as duas mãos \\
& Atirar bola de $20 \mathrm{~cm}$ na parede, acima da cabeça, e agarrar \\
& Repicar bola de tênis no chão e agarrar com as duas mãos \\
& Repicar bola de tênis no chão e agarrar com a mão de preferência \\
\hline d4455 (apanhar) & Agarrar saco de areia \\
& Repicar bola de 20 cm no chão e agarrar com as duas mãos \\
& Atirar bola de 20 cm na parede, acima da cabeça, e agarrar \\
& Repicar bola de tênis no chão e agarrar com as duas mãos \\
& Repicar bola de tênis no chão e agarrar com a mão de preferência \\
& Agarrar uma bola de tênis com as duas mãos \\
& Agarrar uma bola de tênis com a mão de preferência \\
& Equilibrista tandem \\
\hline d450 (andar) & Salto do coelho \\
& Jogo da amarelinha: com 5 padrões \\
d4553 (saltar) & Polichinelo simples \\
& Polichinelo simples \\
& Agarrar uma bola de tênis com as duas mãos \\
& Agarrar uma bola de tênis com a mão de preferência \\
\hline d9200 (jogos) & \\
& \\
\hline
\end{tabular}

Fonte: Dados da pesquisa.

ambientais). No componente $b$ (funções do corpo), os itens do Questionário de Pais foram correlacionados a categorias dos capítulos 1 (Funções mentais) e 7 (Funções neuromusculoesqueléticas e relacionadas ao movimento); no componente $d$ (atividades e participação), a categorias de todos os capítulos (1 a 9); e no componente $e$ (fatores ambientais) apenas a categorias do capítulo 3 (Apoio e relacionamentos). Seis itens foram codificados como fp (fatores pessoais) e um item foi marcado como nc (não coberto pela CIF). Não houve itens codificados com o componente $s$ (estrutura do corpo). 0 Quadro 2 apresenta a codificação completa de todos os itens do Questionário de Pais da Acoordem.

Os 41 itens do Questionário de Professores foram relacionados em 29 categorias, de 9 capítulos da CIF, dos componentes $b$ (funções do corpo) e $d$ (atividades e participação). Não houve itens codificados com os componentes e (fatores ambientais) e $s$ (estrutura do corpo). Dois itens foram codificados como fp (fatores pessoais) e outros dois como nc (não coberto pela CIF). No componente $b$ (funções do cor$p o$ ), houve codificação de categorias apenas no capítulo 1 (Funções mentais), enquanto no componente $d$ (atividades e participação) os itens foram correlacionados a categorias de quase todos os capítulos. 0 único capítulo que não apresentou nenhuma categoria correlacionada a algum item do Questionário de Professores foi o capítulo 8 (Áreas principais $d a$ vida). A codificação dos itens do Questionário de Professores é apresentada no Quadro 3.

\section{Discussão}

O desenvolvimento de regras para correlacionar os itens de uma medida de desfecho com a CIF forneceu um procedimento padronizado por meio do qual as medidas disponíveis em saúde e reabilitação podem ser examinadas, considerando a 
Quadro 2 - Codificação dos itens do Questionário de Pais de acordo com a CIF

(Continua)

\begin{tabular}{|c|c|c|}
\hline Componente da CIF & Categoria da CIF & Item da Acoordem \\
\hline \multirow{8}{*}{ Funções do corpo } & b1263 (estabilidade psíquica) & $\begin{array}{l}\text { Tem dificuldade para aceitar mudanças na rotina, faz birra ou } \\
\text { fica irritado(a) quando acontece alguma coisa diferente ou } \\
\text { inesperada }\end{array}$ \\
\hline & b140 (funções da atenção) & $\begin{array}{l}\text { É difícil andar com a criança na rua, pois ele(a) pode correr } \\
\text { e atravessar a rua, sem atentar para o perigo }\end{array}$ \\
\hline & & $\begin{array}{l}\text { Inquieto, movimenta ou mexe muito o corpo (ex: balança, } \\
\text { cruza e descruza as pernas) }\end{array}$ \\
\hline & b147 (funções psicomotoras) & Agitado, muda de atividade a cada 5-10 minutos \\
\hline & & $\begin{array}{l}\text { Troca de mãos quando usa o lápis (sem preferência, usa } \\
\text { mão direita ou esquerda) }\end{array}$ \\
\hline & & Cansa-se facilmente em atividade físicas \\
\hline & $\begin{array}{l}\text { b7401 (resistência de grupos } \\
\text { musculares) }\end{array}$ & $\begin{array}{l}\text { Mostra sinais de cansaço, mesmo em trabalhos curtos } \\
\text { de escrita (ex: balança as mãos, paradas para descanso, } \\
\text { lentidão) }\end{array}$ \\
\hline & $\begin{array}{l}\text { b770 (funções relacionadas com } \\
\text { o padrão da marcha) }\end{array}$ & Anda com boa postura \\
\hline \multirow{18}{*}{ Atividades e participação } & d130 (imitar) & Copia material escrito correta e legivelmente \\
\hline & $\begin{array}{l}\text { d1551 (adquirir competências } \\
\text { complexas) }\end{array}$ & $\begin{array}{l}\text { Aprende tarefas motoras novas com facilidade (ex: andar de } \\
\text { bicicleta, nadar) }\end{array}$ \\
\hline & \multirow{6}{*}{ d170 (escrever) } & Escreve com facilidade \\
\hline & & Deixa espaços apropriados entre letras e palavras \\
\hline & & Escreve letras e palavras legíveis \\
\hline & & Escreve números legíveis \\
\hline & & Escreve com velocidade apropriada \\
\hline & & $\begin{array}{l}\text { Escreve com pressão adequada do lápis (nem forte nem } \\
\text { leve demais) }\end{array}$ \\
\hline & $\begin{array}{l}\text { d2101 (realizar uma tarefa } \\
\text { complexa) }\end{array}$ & Faz o "para-casa" no tempo previsto \\
\hline & d230 (realizar a rotina diária) & $\begin{array}{l}\text { Guarda corretamente os materiais escolares na pasta } \\
\text { ou mochila }\end{array}$ \\
\hline & d2300 (seguir rotinas) & Segue as rotinas da casa \\
\hline & d2302 (concluir a rotina diária) & $\begin{array}{l}\text { Obedece horários específicos para estudo, brincadeira, TV, } \\
\text { banho, dormir }\end{array}$ \\
\hline & d332 (cantar) & Cantar e representar \\
\hline & $\begin{array}{l}\text { d3350 (produzir mensagens } \\
\text { usando linguagem corporal) }\end{array}$ & Cantar e representar \\
\hline & $\begin{array}{l}\text { d3352 (produzir mensagens } \\
\text { usando desenhos e fotografias) }\end{array}$ & Desenhar, pintar e colorir \\
\hline & d415 (manter a posição do corpo) & Tropeça, cai, propenso a acidentes \\
\hline & d4153 (permanecer sentado) & Mantém boa postura sentado(a) \\
\hline & d4351 (dar pontapés) & Chuta bola com facilidade \\
\hline
\end{tabular}


Quadro 2 - Codificação dos itens do Questionário de Pais de acordo com a CIF

(Continua)

\begin{tabular}{|c|c|c|}
\hline Componente da CIF & Categoria da CIF & Item da Acoordem \\
\hline \multirow{24}{*}{ Atividades e participação } & d440 (movimentos finos da mão) & $\begin{array}{l}\text { Abre e coloca pasta dental na escova } \\
\text { Tromba, quebra brinquedos, objetos escapam das mãos } \\
\text { É habilidoso(a) para fazer trabalhos manuais } \\
\text { Recorta papel com tesoura com facilidade }\end{array}$ \\
\hline & d4454 (atirar) & $\begin{array}{l}\text { Acerta bola em alvo ou joga para outra pessoa com } \\
\text { facilidade }\end{array}$ \\
\hline & d4455 (apanhar) & Agarra bola com facilidade \\
\hline & $\begin{array}{l}\text { d449 (transportar, mover } \\
\text { e manusear objetos, } \\
\text { outros especificados e não } \\
\text { especificados) }\end{array}$ & Coloca comida no prato \\
\hline & d450 (andar) & Anda com boa postura \\
\hline & d4551 (subir/descer) & Sobe e desce escadas com facilidade \\
\hline & d4552 (correr) & Corre com facilidade \\
\hline & d4553 (saltar) & $\begin{array}{l}\text { Salta ou pula obstáculos com facilidade } \\
\text { Pula corda com facilidade }\end{array}$ \\
\hline & d4554 (nadar) & Nadar \\
\hline & d470 (utilização de transporte) & Entra e sai do ônibus ou do carro com facilidade \\
\hline & d5100 (lavar partes do corpo) & Lava e seca as mãos e o rosto \\
\hline & d5101 (lavar todo o corpo) & Toma banho \\
\hline & d5102 (secar-se) & Lava e seca as mãos e o rosto \\
\hline & d5201 (cuidar dos dentes) & Escova os dentes \\
\hline & $\begin{array}{l}\text { d5202 (cuidar do cabelo e da } \\
\text { barba) }\end{array}$ & Penteia e/ou escova os cabelos \\
\hline & $\begin{array}{l}\text { d530 (cuidados relacionados com } \\
\text { os processos de excreção) }\end{array}$ & Limpa-se após o uso do banheiro \\
\hline & d540 (vestir-se) & Abre e fecha zíper e botões \\
\hline & d5400 (vestir roupa) & Veste todas as peças de roupa (ex: uniforme escolar) \\
\hline & d5401 (despir roupa) & Tira todas as peças de roupa (ex: para tomar banho) \\
\hline & d5402 (calçar) & $\begin{array}{l}\text { Calça sapatos de diferentes modelos (ex: tênis, sandália, } \\
\text { mocassim) }\end{array}$ \\
\hline & $\begin{array}{l}\text { d5404 (escolha de roupa } \\
\text { apropriada) }\end{array}$ & Escolhe as próprias roupas no armário ou gaveta \\
\hline & d550 (comer) & Usa faca para cortar alimentos \\
\hline & d560 (beber) & Serve água de jarro ou garrafa \\
\hline & $\begin{array}{l}\text { d640 (realizar as tarefas } \\
\text { domésticas) }\end{array}$ & Guarda roupas no armário ou gavetas \\
\hline
\end{tabular}


Quadro 2 - Codificação dos itens do Questionário de Pais de acordo com a CIF

(Continua)

\begin{tabular}{|c|c|c|}
\hline Componente da CIF & Categoria da CIF & Item da Acoordem \\
\hline \multirow{32}{*}{ Atividades e participação } & \multirow{3}{*}{$\begin{array}{l}\text { d7203 (interagir de acordo com } \\
\text { as regras sociais) }\end{array}$} & $\begin{array}{l}\text { Tem bom comportamento em restaurantes, locais públicos } \\
\text { e festas }\end{array}$ \\
\hline & & $\begin{array}{l}\text { Tem bom comportamento em locais onde precisa ficar } \\
\text { quieto por mais tempo (ex: igreja, teatro) }\end{array}$ \\
\hline & & $\begin{array}{l}\text { Fica extremamente agitado(a) em festas, acabando por } \\
\text { brigar, chorar ou precisar ir embora mais cedo, em razão do } \\
\text { comportamento }\end{array}$ \\
\hline & $\begin{array}{l}\text { d750 (relacionamentos sociais } \\
\text { informais) }\end{array}$ & $\begin{array}{l}\text { Tem bom comportamento quando visita colegas, sem } \\
\text { supervisão dos pais }\end{array}$ \\
\hline & $\begin{array}{l}\text { d7601 (relacionamentos entre } \\
\text { pais e filhos) }\end{array}$ & $\begin{array}{l}\text { Quando acompanha os pais ao shopping center ou } \\
\text { supermercado colabora e é paciente para esperar }\end{array}$ \\
\hline & d820 (educação escolar) & Faz o "para-casa" no tempo previsto \\
\hline & \multirow{4}{*}{ d920 (recreação e lazer) } & $\begin{array}{l}\text { Usa brinquedos móveis, como balanços e gangorra, com } \\
\text { facilidade }\end{array}$ \\
\hline & & $\begin{array}{l}\text { É um dos últimos a ser escolhido para compor times em } \\
\text { esportes ou atividades motoras }\end{array}$ \\
\hline & & Brinca de maneira organizada \\
\hline & & Assiste à televisão \\
\hline & \multirow{15}{*}{ d9200 (jogos) } & Pegador/esconde-esconde \\
\hline & & Amarelinha \\
\hline & & Outras brincadeiras com bola \\
\hline & & Nadar \\
\hline & & Pula-corda, elástico \\
\hline & & Bicicleta, skate e patinete, patins \\
\hline & & Parque (escorregador, balanço) \\
\hline & & Boneca/bonecos de ação \\
\hline & & Casinha, comidinha, mamãe e filhinha, escolinha \\
\hline & & Jogos de construção/Lego® \\
\hline & & Quebra-cabeça \\
\hline & & Videogame \\
\hline & & Bolinha de gude \\
\hline & & Soltar pipa \\
\hline & & Jogos de mesa/baralho \\
\hline & \multirow{3}{*}{ d9201(esportes) } & Futebol \\
\hline & & Vôlei ou basquete \\
\hline & & Peteca, tênis, jogos com raquete \\
\hline & \multirow{4}{*}{ d9202 (arte e cultura) } & Cantar e representar \\
\hline & & Dançar, aulas de dança \\
\hline & & Tocar instrumento musical \\
\hline & & Ler livros e revistinhas \\
\hline Fatores ambientais & $\begin{array}{l}\text { e325 (conhecidos, pares, } \\
\text { colegas, vizinhos e membros da } \\
\text { comunidade) }\end{array}$ & $\begin{array}{l}\text { Os colegas chamam para brincar ou participar de } \\
\text { brincadeiras e/ou atividades físicas }\end{array}$ \\
\hline
\end{tabular}


Cardoso AA, Magalhães LC, de Lacerda TTB, Andrade PMO.

Quadro 2 - Codificação dos itens do Questionário de Pais de acordo com a CIF

(Conclusão)

\begin{tabular}{lll}
\hline Componente da CIF & Categoria da CIF & Item da Acoordem \\
\hline Fatores ambientais & e310 (família próxima) & $\begin{array}{l}\text { A família restringe suas atividades sociais em razão do } \\
\text { comportamento da criança }\end{array}$ \\
\hline & $\begin{array}{l}\text { Gosta de participar de atividades físicas ou esportivas } \\
\text { Desiste facilmente de atividades motoras mais difíceis } \\
\text { Recusa-se a fazer trabalhos que envolvem escrever, } \\
\text { desenhar e colorir } \\
\text { Prefere brincadeiras mais paradas (ex: ler, contar histórias) } \\
\text { Fatores pessoais }\end{array}$ & $\begin{array}{l}\text { Prefere brincar sentado ou mesmo deitado } \\
\text { Prefere brincar com crianças bem mais jovens }\end{array}$ \\
& Prefere brincar sozinho \\
\hline Não cobertos pela CIF & É prazeroso ir com a criança à praia, ao clube ou ao parque, \\
& pois ele(a) se comporta apropriadamente \\
\hline \multirow{2}{*}{ NC } &
\end{tabular}

Fonte: Dados da pesquisa.

Quadro 3 - Codificação dos itens do Questionário de Professores de acordo com a CIF

(Continua)

\begin{tabular}{|c|c|c|}
\hline Componente da CIF & Categoria da CIF & Item da Acoordem \\
\hline \multirow{4}{*}{ Funções do corpo } & b1141 (orientação em relação ao lugar) & $\begin{array}{l}\text { Anda pela escola sem se perder, reconhece a localização } \\
\text { da sala de aula, do banheiro e outros pontos importantes } \\
\text { para mobilidade na escola }\end{array}$ \\
\hline & b1400 (manutenção da atenção) & Mantém atenção durante as tarefas \\
\hline & \multirow{2}{*}{ b147 (funções psicomotoras) } & $\begin{array}{l}\text { Inquieto, se move muito na carteira, sempre mexendo } \\
\text { com alguma coisa }\end{array}$ \\
\hline & & $\begin{array}{l}\text { Troca de mãos quando usa o lápis (sem preferência, usa } \\
\text { mão direita ou esquerda) }\end{array}$ \\
\hline \multirow{11}{*}{ Atividades e participação } & \multirow{2}{*}{ d170 (escrever) } & Escreve com facilidade \\
\hline & & Deixa espaços apropriados entre letras e palavras \\
\hline & \multirow{3}{*}{ d170 (escrever) } & Escreve números, letras e palavras legíveis \\
\hline & & $\begin{array}{l}\text { Escreve com pressão adequada no lápis (nem forte nem } \\
\text { leve demais) }\end{array}$ \\
\hline & & Escreve com velocidade apropriada \\
\hline & d2101 (realizar uma tarefa complexa) & $\begin{array}{l}\text { Se prepara para as tarefas selecionando caderno, livro } \\
\text { ou materiais apropriados }\end{array}$ \\
\hline & d2200 (realizar tarefas múltiplas) & Passa de uma atividade para outra de forma organizada \\
\hline & $\begin{array}{l}\text { d2204 (completar tarefas múltiplas } \\
\text { independentemente) }\end{array}$ & $\begin{array}{l}\text { Trabalha de maneira independente para concluir os } \\
\text { trabalhos solicitados }\end{array}$ \\
\hline & d230 (realizar a rotina diária) & $\begin{array}{l}\text { Maneja a pasta/mochila, retirando e colocando livros } \\
\text { e materiais }\end{array}$ \\
\hline & d2301 (gerir a rotina diária) & Mantém material escolar organizado na carteira \\
\hline & $\begin{array}{l}\mathrm{d} 310 \text { (comunicar e receber } \\
\text { mensagens orais) }\end{array}$ & Entende as regras da sala de aula \\
\hline
\end{tabular}


Quadro 3 - Codificação dos itens do Questionário de Professores de acordo com a CIF

(Continua)

\begin{tabular}{|c|c|c|}
\hline Componente da CIF & Categoria da CIF & Item da Acoordem \\
\hline \multirow{20}{*}{ Atividades e participação } & d330 (falar) & Expressa-se verbalmente, fala compreensível \\
\hline & $\begin{array}{l}\text { d3352 (produzir mensagens usando } \\
\text { desenhos e fotografia) }\end{array}$ & Colore e desenha com facilidade \\
\hline & d4153 (permanecer sentado) & $\begin{array}{l}\text { Mantém boa postura quando sentado(a) na carteira } \\
\text { Fica muito inclinado/deitado sobre a carteira ou com a } \\
\text { cabeça apoiada na mão }\end{array}$ \\
\hline & d4301 (transportar nas mãos) & $\begin{array}{l}\text { Transporta materiais, livros e cadernos pela sala sem } \\
\text { trombar nas carteiras }\end{array}$ \\
\hline & d440 (movimentos finos da mão) & $\begin{array}{l}\text { Recorta com tesoura com facilidade } \\
\text { É habilidoso(a) nas aula de artes ou de trabalhos } \\
\text { manuais } \\
\text { Abre/fecha merendeira, embalagens e alimentos com } \\
\text { facilidade }\end{array}$ \\
\hline & d450 (andar) & $\begin{array}{l}\text { Anda e corre com boa postura } \\
\text { Anda pela escola sem se perder, reconhece a localização } \\
\text { da sala de aula, do banheiro e outros pontos importantes } \\
\text { para mobilidade na escola }\end{array}$ \\
\hline & d4501 (andar distâncias longas) & $\begin{array}{l}\text { Acompanha o ritmo da turma em passeios ou } \\
\text { caminhadas }\end{array}$ \\
\hline & d4503 (andar contornando obstáculos) & $\begin{array}{l}\text { Transporta materiais, livros e cadernos pela sala, sem } \\
\text { trombar nas carteiras }\end{array}$ \\
\hline & d4551 (subir/descer) & Sobe e desce escadas com facilidade \\
\hline & d4552 (correr) & Anda e corre com boa postura \\
\hline & d4553 (saltar) & Pula ou salta obstáculos com facilidade \\
\hline & $\begin{array}{l}\text { d530 (cuidados relacionados com os } \\
\text { processos de excreção) }\end{array}$ & Retira e coloca peças de roupa para uso do banheiro \\
\hline & d650 (cuidar dos objetos da casa) & $\begin{array}{l}\text { Mantém seus pertences organizados e prontos para uso } \\
\text { no espaço designado }\end{array}$ \\
\hline & \multirow{2}{*}{$\begin{array}{l}\text { d7203 (interagir de acordo com as } \\
\text { regras sociais) }\end{array}$} & $\begin{array}{l}\text { Aguarda sua vez para falar, de acordo com as regras da } \\
\text { sala (ex: levanta a mão, não interrompe os outros) }\end{array}$ \\
\hline & & $\begin{array}{l}\text { Apresenta comportamento adequado em passeios, } \\
\text { excursões ou outras atividades extraclasse }\end{array}$ \\
\hline & $\begin{array}{l}\text { d7400 (relacionamentos com } \\
\text { superiores) }\end{array}$ & Segue instruções verbais ou solicitações da professora \\
\hline & $\begin{array}{l}\text { d7504 (relacionamentos informais } \\
\text { com pares) }\end{array}$ & $\begin{array}{l}\text { Se dá bem com os colegas } \\
\text { É cooperativo(a) e colabora com os colegas em } \\
\text { trabalhos grupais }\end{array}$ \\
\hline & d820 (educação escolar) & Segue instruções verbais ou solicitações da professora \\
\hline & \multirow{2}{*}{ d920 (recreação e lazer) } & $\begin{array}{l}\text { Usa brinquedos móveis, como balanços e gangorra, } \\
\text { com facilidade }\end{array}$ \\
\hline & & $\begin{array}{l}\text { Participa de brincadeiras com bola e atividades motoras } \\
\text { durante o recreio }\end{array}$ \\
\hline
\end{tabular}


Quadro 3 - Codificação dos itens do Questionário de Professores de acordo com a CIF

(Conclusão)

\begin{tabular}{lll}
\hline Componente da CIF & Categoria da CIF & Item da Acoordem \\
\hline Atividades e participação & d9201 (esportes) & Participa com facilidade das aulas de educação física \\
\hline Fatores pessoais & FP & $\begin{array}{l}\text { Recusa-se a fazer trabalhos que envolvem escrever, } \\
\text { desenhar e colorir } \\
\text { Gosta de participar de atividades físicas e esportivas }\end{array}$ \\
\hline Não cobertos pela CIF & NC & $\begin{array}{l}\text { A letra é boa ou compatível com as demandas da } \\
\text { professora } \\
\text { Tem dificuldade para passar de atividades mais agitadas } \\
\text { para atividades mais calmas }\end{array}$ \\
\hline
\end{tabular}

Fonte: Dados da pesquisa.

perspectiva da funcionalidade (33). A Acoordem foi criada com o objetivo de possibilitar a detecção de atraso motor em crianças brasileiras de 4 a 8 anos de idade, dentro da perspectiva de avaliar os componentes motores em associação com habilidades relevantes para o desempenho funcional em casa e na escola. Para tanto, o instrumento conta com itens de observação direta da criança e dois questionários (Questionário de Pais e Questionário de Professores).

Todos os itens de observação direta da criança puderam ser codificados e correlacionados à CIF, sendo a maioria correlacionada ao componente $d$ (atividades e participação). Nos questionários de pais e professores também foi possível codificar e correlacionar os itens com a CIF, com exceção de um item no Questionário de Pais (É prazeroso ir com a criança à praia, ao clube ou ao parque, pois ela se comporta apropriadamente) e dois itens no Questionário de Professores (A letra é boa ou compatível com as demandas da professora e Tem dificuldade para passar de atividades mais agitadas para mais calmas). Em ambos os questionários foi possível observar que a maioria dos itens também foi correlacionada a categorias do componente $d$ (atividades e participação). Observa-se, então, que a maioria dos itens da Acoordem avalia os componentes motores em associação com habilidades funcionais relevantes para o desempenho em casa e na escola, o que é um ponto positivo do instrumento quando se considera a perspectiva biopsicossocial proposta pela CIF (6).

A proximidade entre os itens da Acoordem e a CIF pode ser parcialmente explicada pelo fato de que a criação da Acoordem foi norteada pelo modelo da
CIF (6), ou seja, o objetivo do instrumento é detectar, além do atraso motor, as consequências funcionais na vida das crianças. 0 conteúdo da Acoordem, no entanto, não inclui itens correlacionados ao componente $s$ (estrutura do corpo) da CIF. A ausência de itens codificados com esse componente pode ser atribuída ao fato de que o objetivo da Acoordem não é informar sobre uma condição de saúde, mas sim mensurar aspectos relacionados à funcionalidade das crianças.

A OMS reconheceu a importância de distinguir, quantificar e explicar a influência de fatores pessoais nos processos de incapacidade e funcionalidade (19). Embora a CIF ainda não tenha códigos específicos para tais fatores, as regras de codificação propostas por Cieza et al. (19) permitem a identificação de fatores pessoais. Oito itens dos questionários (seis itens no Questionário de Pais e dois itens no Questionário de Professores) foram codificados com fp (fatores pessoais). A inclusão de fatores pessoais na Acoordem reforça a capacidade do instrumento de mensurar os construtos de funcionalidade e saúde.

De acordo com o modelo da CIF, fatores ambientais podem influenciar positiva ou negativamente a funcionalidade, incapacidade e saúde humanas, servindo como facilitadores ou barreiras $(1,5-7)$. Na Acoordem, apenas o Questionário de Pais apresentou itens codificados como $e$ (fatores ambientais). 0 fato de apenas dois itens da Acoordem informarem sobre fatores ambientais pode ser uma limitação do conteúdo do instrumento, uma vez que tal informação é essencial no processo de avaliação (6). Simeonsson et al. (15) apontam que, para definir a 
influência de fatores ambientais, o nicho de desenvolvimento é caracterizado por três componentes: o ambiente físico e social em que a criança vive; os hábitos de cuidado e educação da criança; e a psicologia dos cuidadores, isto é, os valores e crenças que definem as práticas dos pais e cuidadores. Os dois itens da Acoordem codificados como fatores ambientais (Os colegas chamam para brincar ou participar de brincadeiras e/ou atividades físicas e $A$ família restringe suas atividades sociais em razão do comportamento da criança) contemplam o primeiro componente apontado por Simeonsson et al. (15). 0 conteúdo da Acoordem não contempla os hábitos de cuidado e educação da criança nem a psicologia dos cuidadores. Observa-se, no entanto, que, como teste focado no desempenho, a inclusão de itens nessas áreas poderia tornar o instrumento excessivamente longo, podendo outros instrumentos ser utilizados em associação com a Acoordem para uma avaliação mais completa.

Os resultados do presente estudo demonstraram que, de modo geral, os itens da Acoordem puderam ser codificados e relacionados à estrutura da CIF, demonstrando que o instrumento segue a tendência atual de avaliar os componentes motores em associação com habilidades relevantes para o desempenho funcional em casa e na escola.

\section{Conclusão}

Os resultados deste estudo ajudaram a documentar que o conteúdo específico da Acoordem é compatível com a perspectiva biopsicossocial da OMS. Além disso, os resultados mostraram que a Acoordem pode beneficiar pesquisadores e clínicos da área de reabilitação, uma vez que o instrumento oferece a oportunidade para obter informações que vão além da perspectiva do modelo biomédico tradicional, que tende a dar atenção exclusiva às estruturas e funções do corpo. A Acoordem ajuda o terapeuta a contextualizar a avaliação conforme a realidade funcional/ambiental da criança, por meio da investigação tanto das capacidades como do desempenho em atividades e participação em casa e na escola, com base na observação dos pais e professores, feita no contexto diário da criança. Uma limitação observada no conteúdo do instrumento é o fato de apenas dois itens informarem sobre fatores ambientais. Apesar da limitação, o uso da Acoordem por fisioterapeutas e terapeutas ocupacionais poderá ajudar a sistematizar a avaliação motora e operacionalizar ações de promoção do desempenho funcional e da participação nos diferentes contextos, em parceria com os pais e professores.

\section{Referências}

1. Farias M, Buchalla CM. Classificação Internacional de Funcionalidade, Incapacidade e Saúde: conceitos, usos e perspectivas. Rev Bras Epidemiol. 2005; 8(2): 187-93.

2. Sampaio RF, Mancini MC, Gonçalves GGP, Bittencourt NFN, Miranda AD, Fonseca ST. Aplicação da Classificação Internacional de Funcionalidade, Incapacidade e Saúde (CIF) na prática clínica do fisioterapeuta. Rev Bras Fisioter. 2005;9(2):129-36.

3. Di Nubila HBV. Aplicação das classificações CID-10 e CIF nas definições de deficiência e incapacidade. [tese]. São Paulo: Faculdade de Saúde Pública da Universidade de São Paulo; 2007.

4. Di Nubila HBV, Buchalla CM. O papel das classificações da OMS - CID e CIF nas definições de deficiência e incapacidade. Rev Bras Epidemiol. 2008;11(2): 324-35.

5. Organização Mundial da Saúde. Rumo a uma linguagem comum para funcionalidade incapacidade e saúde - CIF. Genebra: OMS; 2002.

6. Organização Mundial da Saúde. Organização Panamericana de Saúde. Classificação Internacional de Funcionalidade, Incapacidade e Saúde (CIF). São Paulo: Edusp; 2003.

7. Masala C, Petretto DR. From disablement to enablement: conceptual models of disability in the 20th century. Disabil Rehabil. 2008;30(17):1233-44.

8. Üstun TB, Chatterji S, Bickenback J, Kostanjsek N, Schneider M. The international classification of functioning, disability and health: a new tool for understanding disability and health. Disabil Rehabil. 2003; 25(11-12):565-71.

9. Drumond AS. Exploração do Disabilities, Arm, Shoulder and Hand (DASH) através da Classificação Internacional de Funcionalidade, Incapacidade e Saúde (CIF) e da Análise Rasch. [dissertação]. Belo Horizonte: Universidade Federal de Minas Gerais; 2006. 
10. Mângia EF, Muramoto M, Lancman S. Classificação Internacional de Funcionalidade, Incapacidade e Saúde (CIF): processo de elaboração e debate sobre a questão da incapacidade. Rev Ter Ocup USP. 2008;19(2):121-30.

11. Andrade PMO. Avaliação do estágio da fisioterapia conforme as diretrizes curriculares e a perspectiva biopsicossocial da organização mundial de saúde. Avaliação (Campinas). 2010;15(2):121-34.

12. World Health Organization. WHO international classification of functioning, disability and health version for children and youth. Geneva: WHO; 2004.

13. McDougall J, Miller L. Measuring chronic health condition and disability as distinct concepts in national surveys of school-aged children in Canada: a comprehensive review with recommendations based on the ICD-10 and ICF. Disabil Rehabil. 2003;25(16):922-39.

14. McConachie H, Colver AF, Forsyth RJ, Jarvis SN, Parkinson KN. Participation of disabled children: how should it be characterized and measured? Disabil Rehabil. 2006;28(18):1157-64.

15. Simeonsson RJ, Leonardi M, Lollars D, BjorckAkesson E, Hollenweger J, Martinuzzi A. Applying the International Classification of Functioning, Disability and Health (ICF) to measure childhood disability. Disabil Rehabil. 2003;25(11-12):602-10.

16. Buñuales MTJ, Diego PG, Moreno JMM. La Clasificación Internacional del Funcionamiento, de la Discapacidad y de la Salud (CIF) 2001. Rev Esp Salud Publica. 2002;76(4):271-9.

17. Di Nubila HBV. Uma introdução à CIF - Classificação Internacional de Funcionalidade, Incapacidade e Saúde. Rev Bras Saúde Ocup. 2010;35(121):122-3.

18. Stucki G, Ewert T, Cieza A. Value and application of the ICF in rehabilitation medicine. Disabil Rehabil. 2002;24(7):932-38.

19. Cieza A, Geugh S, Chatterji S, Kostanjsek N, Üstün B, Stucki G. ICF linking rules: an update based on lessons learned. J Rehabil Med. 2005;37(4):212-8.

20. Geyh S, Kurt T, Brockow T, Cieza A, Ewert T, Omar Z, et al. Identifying the concepts contained in outcome measures of clinical trials on stroke using the International Classification of Functioning, Disability and Health as a reference. J Rehabil Med. 2004;(44 Suppl):56-62.
21. Cieza A, Stucki G. Content comparison of health-related quality of life (HRQOL) instruments based on the international classification of functioning, disability and health (ICF). Qual Life Res. 2005;14(5):1225-37.

22. Geyh S, Cieza A, Kollerits B, Grimby G, Stucki G. Content comparison of health-related quality of life measures used in stroke based on the international classification of functioning, disability and health (ICF): a systematic review. Qual Life Res. 2007;16(5): 833-51.

23. Stamm TA, Cieza A, Coenen M, Machold KP, Nell VP, Smolen JS, et al. Validating the international classification of functioning, disability and health comprehensive core set for rheumatoid arthritis from the patient perspective: a qualitative study. Arthritis Rheum. 2005;53(3):431-9.

24. Coenen M, Cieza A, Stamm TA, Amann E, Kollerits B, Stucki G. Validation of the international classification of functioning, disability and health (ICF) core set for rheumatoid arthritis from the patient perspective using focus groups. Arthritis Res Ther. 2006;8(4):1-14.

25. Magalhães LC, Rezende MB, Cardoso AA. Avaliação da coordenação e destreza motora - ACOORDEM. Versão Experimental III. [tese]. Belo Horizonte: Universidade Federal de Minas Gerais; 2008.

26. Magalhães LC, Nascimento VCS, Rezende MB. Avaliação da coordenação e destreza motora - ACOORDEM; Etapas de criação e perspectivas de validação. Rev Ter Ocup USP. 2004;15(1):17-25.

27. Larkin D, Cermak SA. Issues in identification and assessment of Developmental Coordination Disorder. In: Cermak S, Larkin D. (Ed.). Developmental coordination disorder. Albany, New York: Delmar Thompson Learning; 2002. p. 86-102.

28. Missiuna C, Rivard L, Bartlett D. Exploring assessment tools and the target of intervention for children with Developmental Coordination Disorder. Phys Occup Ther Pediatr. 2006:26(1/2):71-89.

29. Cury RLSM. Criação de um protocolo de avaliação do equilíbrio corporal em crianças de 4, 6 e 8 anos de idade: uma perspectiva funcional. [dissertação]. Belo Horizonte: Universidade Federal de Minas Gerais, Programa de Mestrado em Ciência da Reabilitação, Escola de Educação Física, Fisioterapia e Terapia Ocupacional; 2005. 
30. Cardoso AA, Magalhães LC. Bilateral coordination and motor sequencing in Brazilian children: preliminary construct validity and reliability analysis. Occup Ther Int. 2009;16(2):107-21.

31. Lacerda TB, Magalhães LC. Validade de conteúdo de questionários de coordenação motora para pais e professores. Rev Ter Ocup USP. 2007;18(2):63-77.

32. Cardoso AA, Magalhães LC, Galvão BAP. Fine motor coordination and manual dexterity of the assessment of motor coordination and dexterity (AMCD). In: 15th International Congress of the World Federation of Occupational Therapists. Santiago - Chile: WFOT; 2010. p. 1744.
33. Drumond AS, Sampaio RF, Mancini MC, Kirkwood RN, Stamm TA. Linking the disabilities of arm, shoulder and hand to the international classification of functioning, disability and health. J Hand Ther. 2007; 20(4):336-43.

Recebido: 29/09/2010

Received: 09/29/2010

Aprovado: 25/05/2011

Approved: 05/25/2011 